\title{
Intensive Imaging-based Follow-up of Surgically Treated Localised Renal Cell Carcinoma Does Not Improve Post-recurrence Survival: Results from a European Multicentre Database (RECUR)
}

Saeed Dabestani ${ }^{\text {a, }}{ }^{,}$, Christian Beisland ${ }^{\text {b,c, },}$, Grant D. Stewart ${ }^{\mathrm{d}}$, Karim Bensalah ${ }^{\mathrm{e}}$, Eirikur Gudmundsson $^{\mathrm{f}}$, Thomas B. Lam ${ }^{\text {g,h }}$, William Gietzmann ${ }^{\mathrm{g}}$, Paimaun Zakikhani ${ }^{\mathrm{h}}$, Lorenzo Marconi i , Sergio Fernandéz-Pello ${ }^{\mathrm{j}}$, Serenella Monagas ${ }^{\mathrm{k}}$, Samuel Paul Williams ${ }^{1}$, Christian Torbrand $^{\mathrm{a}}$, Thomas Powles ${ }^{\mathrm{m}}$, Erik Van Werkhoven ${ }^{\mathrm{n}}$, Richard Meijer ${ }^{\mathrm{o}}$, Alessandro Volpe ${ }^{\mathrm{p}}$, Michael Staehler ${ }^{\mathrm{q}}$, Börje Ljungberg ${ }^{\mathrm{r}}$, Axel Bex ${ }^{\mathrm{s}, *}$

${ }^{a}$ Department of Clinical Sciences, Lund University, Skane University Hospital, Lund, Sweden

${ }^{\mathrm{b}}$ Department of Urology, Haukeland University Hospital, Bergen, Norway

${ }^{c}$ Department of Clinical Medicine, University of Bergen, Bergen, Norway

d Academic Urology Group, Department of Surgery, University of Cambridge, Cambridge, UK

${ }^{\mathrm{e}}$ Department of Urology, University of Rennes, Rennes, France

${ }^{\mathrm{f}}$ Department of Urology, Landspitali University Hospital, Reykjavik, Iceland

${ }^{g}$ Academic Urology Unit, University of Aberdeen, Aberdeen, UK

${ }^{\mathrm{h}}$ Department of Urology, Aberdeen Royal Infirmary, Aberdeen, UK

${ }^{i}$ Department of Urology, Coimbra University Hospital, Coimbra, Portugal

${ }^{\mathrm{j}}$ Department of Urology, Cabueñes University Hospital, Gijón, Spain

${ }^{k}$ Department of Urology, San Agustin University Hospital, Aviles, Spain

${ }^{1}$ Medical School, University of Edinburgh, Edinburgh, UK

${ }^{\mathrm{m}}$ Barts Cancer Institute, Queen Mary University of London, London, UK

${ }^{\mathrm{n}}$ Department of Bioinformatics and Statistics, The Netherlands Cancer Institute, Amsterdam, The Netherlands

${ }^{\circ}$ Department of Urology, University Medical Centre Utrecht, Utrecht, The Netherlands

${ }^{\mathrm{p}}$ Department of Urology, University of Eastern Piedmont, Novara, Italy

${ }^{\mathrm{q}}$ Department of Urology, Klinikum Grosshadern, Ludwig Maximilians University of Munich, Munich, Germany

${ }^{\mathrm{r}}$ Department of Surgical and Perioperative Sciences, Umeå University, Umeå, Sweden

${ }^{\mathrm{s}}$ Division of Surgical Oncology, Department of Urology, The Netherlands Cancer Institute, Amsterdam, The Netherlands

$\dagger$ These authors contributed equally to this paper.

* Corresponding author. Division of Surgical Oncology, Department of Urology, The Netherlands Cancer Institute, Plesmanlaan 121, 1066 CX Amsterdam, The Netherlands. Tel. +3120 5122553; Fax: +31205122554.

E-mail address: a.bex@nki.nl (A. Bex).

Keywords: Kidney cancer; Radical surgery; Follow-up; Imaging; Overall survival

\section{Abstract}


The optimal follow-up (FU) strategy for patients treated for localised renal cell carcinoma (RCC) remains unclear. Using the RECUR database, we studied imaging intensity utilised in contemporary FU to evaluate its association with outcome after detection of disease recurrence. Consecutive patients with nonmetastatic RCC $(n=1612)$ treated with curative intent at 12 institutes across eight European countries between 2006 and 2011 were included. Recurrence occurred in 336 patients. Cross-sectional (computed tomography, magnetic resonance imaging) and conventional (chest X-ray, ultrasound) methods were used in $47 \%$ and 53\%, respectively. More intensive FU imaging (more than twofold) than recommended by the European Association of Urology (EAU) was not associated with improved overall survival (OS) after recurrence. Overall, per patient treated for recurrence remaining alive with no evidence of disease, the number of FU images needed was 542, and 697 for high-risk patients. The study results suggest that use of more imaging during FU than that recommended in the 2017 EAU guidelines is unlikely to improve OS after recurrence. Prospective studies are needed to design optimal FU strategies for the future.

Patient summary: After curative treatment for localised kidney cancer, follow-up is necessary to detect any recurrence. This study illustrates that increasing the imaging frequency during follow-up, even to double the number of follow-up imaging procedures recommended by the European Association of Urology guidelines, does not translate into improved survival for those with recurrence.

The optimal follow-up (FU) strategy for patients treated for localised renal cell carcinoma (RCC) is undetermined. There are controversies regarding modalities and the frequency and timing of imaging. Recent systematic reviews of retrospective studies have concluded that consensus regarding the optimal surveillance is lacking [1,2]. Moreover, it remains unclear if early diagnosis of recurrence improves survival.

The RECUR database is a European Association of Urology (EAU) RCC Guidelines Panel initiative. RECUR is a collaborative multicentre consortium with protocol-based data collection to investigate comparators for evidence-based FU recommendation for localised RCC. The RECUR protocol is available online [3]. In contrast to previously published FU studies, the focus of RECUR is on management and outcomes once a recurrence is detected, rather than from the time of initial treatment with curative intent.

For the present study, RECUR data for 1889 patients with localised RCC from 12 centres (all with appropriate institutional approval) in eight European countries (Supplementary material) were analysed. Eligible patients underwent surgery with curative intent from January 2006 (the start of the tyrosine kinase inhibitor era) to December 2011. After exclusions, the final study population consisted of 1612 patients. Median FU for patients who did not experience recurrence or die during FU was 63 mo (interquartile range 58-76). Patient characteristics are listed in Supplementary Table 1.

The validated risk grouping system described by Leibovich [4] was used for clear cell RCC and the UCLA Integrated Staging System [5] was used for non-clear cell RCC. Overall survival (OS) after recurrence was defined as the time from recurrence until death from any cause or, for patients still alive, to the date of last FU. The total number of imaging procedures per patient was defined as all imaging performed during FU. This was used to calculate the number of imaging procedures needed to identify one patient with recurrent disease, one patient with recurrent disease receiving treatment with curative intent, and one patient with no evidence of disease (NED) following treatment of the recurrence. On the basis of the FU recommendations in the EAU guidelines [6], we calculated the number of images patients within the three risk groups should have undergone until recurrence or last FU. The image ratio (IR) was defined as the total number of imaging scans 
divided by recommended number of imaging scans (Supplementary material). Finally, the correlation between different IR levels and OS after recurrence was investigated. Calculations for image use, cross-sectional imaging, and images needed were based on the total study population $(n=1612)$, while the estimation of OS after recurrence was based on those patients who experienced recurrence and for whom an IR could be established $(n=293$; Supplementary material).

Of 17333 FU imaging procedures performed, 8142 (47\%) were cross-sectional (computed tomography $[\mathrm{CT}]$ or magnetic resonance imaging) and 9191 (53\%) were conventional (chest $\mathrm{X}$-ray or ultrasound). Cross-sectional imaging increased significantly during the study period (Supplementary Fig. 1). Overall, 52 imaging procedures were needed to identify one patient with recurrent disease. Of the 336 patients with recurrences, 92 were treated with curative intent, of whom 32 were alive with NED. The total number of FU imaging procedures needed to identify one patient with a recurrence suitable for treatment with curative intent was 188 . Finally, for one patient who was alive with NED after treatment with curative intent of a recurrence, a total of 542 imaging procedures were required (Table 1). Figure 1 demonstrates similar OS after recurrence for patients with IR of $\leq 0.75,0.76-1.99$, and $\geq 2.0$. For the highrisk group, for which the EAU guidelines recommend 12 imaging procedures (6 CT of the abdomen and $6 \mathrm{CT}$ of the thorax) during $5 \mathrm{yr}$ of $\mathrm{FU}$, we found no OS improvement between patients undergoing $\geq 24$ imaging procedures and those with $\leq 8$ imaging procedures ( $p=$ 0.985). Similar nonsignificant differences were observed for the other risk groups (data not shown).

Imaging in most FU protocols follows defined intervals, with the highest imaging frequency for patients with the highest risk scores. The rationale is that regular imaging has the potential to reveal recurrences early while they are limited and asymptomatic. However, for such imaging strategies, the disease has to behave in a predictable pattern in the majority of patients, with recurrences growing linearly and spreading to distant sites in a predictable fashion. As a higher frequency of imaging was not linked to a better oncological outcome, one might conclude that imaging should be further increased. However, the increase in imaging needed to enhance early detection is not likely to be cost-effective. Furthermore, the biology of RCC may still preclude cure from recurrences in a proportion of patients, regardless of local or systemic treatment options following detection of recurrent lesions, as suggested in the recent TRACERx Renal study by Turajlic et al [7]. Theoretically, more refined and frequent imaging has the potential to introduce a leadtime bias resulting in earlier detection and therapeutic management without changing the outcome.

As the RECUR database is retrospective with its inherent limitations, interpretation requires caution. All centres used their own FU programs with differing intervals for the individual imaging approaches. Therefore, it was not possible to demonstrate to what extent each patient underwent imaging at the recommended time point. Furthermore, we have simplified the EAU recommendation by accepting conventional imaging as equivalent to cross-sectional imaging. We acknowledge that $\mathrm{CT}$ detects recurrence with higher resolution than ultrasound/chest X-ray [8], and that use of conventional imaging might dilute the results obtained. However, in another RECUR report, CT did not show superiority over conventional imaging methods with regard to outcome after detection of recurrences [9]. The fact that we have included all histological subtypes may be a further limitation. However, the major guideline authorities (EAU, American Urological Association, and National Comprehensive Cancer Network) use the same FU recommendation for all RCC subtypes.

A further importance of rationalising FU imaging is cost-effectiveness, as resources are allocated to patients presumed to have been cured that could otherwise be used for diagnosis or treatment. The question of the present cost-effectiveness might be raised, as 542 imaging 
procedures were needed to identify one patient treated for recurrence and being alive with NED.

The present study suggests that a more intensive imaging frequency during FU than that recommended in the 2017 EAU guidelines is unlikely to improve OS after recurrence. Prospective studies are needed to design optimal FU strategies that may be more individualized on the basis of patient and tumour characteristics.

Author contributions: Axel Bex had full access to all the data in the study and takes responsibility for the integrity of the data and the accuracy of the data analysis.

Study concept and design: Dabestani, Beisland, Ljungberg, Stewart, Bex. Acquisition of data: Dabestani, Beisland, Stewart, Gudmundsson, Lam, Gietzmann, Zakikhani, Marconi, Fernandéz-Pello, Monagas, Williams, Torbrand, Meijer, Volpe, Ljungberg, Bex.

Analysis and interpretation of data: Beisland, Dabestani, Bex, Stewart, Ljungberg.

Drafting of the manuscript: Dabestani, Beisland, Ljungberg, Stewart, Bex.

Critical revision of the manuscript for important intellectual content: Dabestani, Beisland, Stewart, Bensalah, Gudmundsson, Lam, Gietzmann, Zakikhani, Marconi, Fernandéz-Pello, Monagas, Williams, Torbrand, Powles, Van Werkhoven, Meijer, Volpe, Staehler, Ljungberg, Bex.

Statistical analysis: Beisland, Dabestani, Van Werkhoven.

Obtaining funding: None.

Administrative, technical, or material support: None.

Supervision: None.

Other: None.

Financial disclosures: Axel Bex certifies that all conflicts of interest, including specific financial interests and relationships and affiliations relevant to the subject matter or materials discussed in the manuscript (eg, employment/affiliation, grants or funding, consultancies, honoraria, stock ownership or options, expert testimony, royalties, or patents filed, received, or pending), are the following: None.

Funding/Support and role of the sponsor: None.

\section{References}

1. Capogrosso P, Capitanio U, La Croce G, et al. Follow-up after treatment for renal cell carcinoma: the evidence beyond the guidelines. Eur Urol Focus 2016;1:272-81.

2. Williamson TJ, Pearson JR, Ischia J, Bolton DM, Lawrentschuk N: Guideline of guidelines: follow-up after nephrectomy for renal cell carcinoma. BJU Int 2016;117:55562.

3. Dabestani S, Beisland C, Stewart GD, et al. Long-term outcomes of follow-up for initially localised clear cell renal cell carcinoma: RECUR database analysis. Eur Urol Focus. In press. http://dx.doi.org/10.1016/j.euf.2018.02.010

4. Leibovich BC, Blute ML, Cheville JC, et al. Prediction of progression after radical nephrectomy for patients with clear cell renal cell carcinoma: a stratification tool for prospective clinical trials. Cancer 2003;97:1663-71.

5. Zisman A, Pantuck AJ, Dorey F, et al. Improved prognostication of renal cell carcinoma using an integrated staging system. J Clin Oncol 2001;19:1649-57. 
6. Ljungberg B, Albiges L, Bensalah K, et al. EAU guidelines on renal cell carcinoma. http://uroweb.org/guideline/renal-cell-carcinoma

7. Turajlic $\mathrm{S}, \mathrm{Xu} \mathrm{H}$, Litchfield $\mathrm{K}$, et al. Tracking cancer evolution reveals constrained routes to metastases: TRACERx Renal. Cell 2018;173:581-594.e512.

8. Doornweerd BH, de Jong IJ, Bergman LM, Ananias HJ. Chest X-ray in the follow-up of renal cell carcinoma. World J Urol 2014;32:1015-9.

9. Beisland C, Dabestani S, Stewart G, et al. Imaging modalities used for follow-up of localized renal cell carcinoma (RCC) and subsequent effect on overall survival after recurrence: RECUR-database analysis. J Clin Oncol 2018;36(6 Suppl):637.

Fig. 1 - Overall survival after recurrence for different image ratio (IR) levels, where IR is the total number of imaging scans divided by the estimated number of imaging scans for the given follow-up period according to European Association of Urology guidelines. There were no significant differences between the groups. 
Table 1 - Number of imaging procedures needed for detection of one patient undergoing TCI for recurrent RCC and for one patient alive with NED after TCI of their recurrence

\begin{tabular}{|c|c|c|c|c|c|c|c|c|}
\hline Risk group & $\begin{array}{l}\text { RCC } \\
\text { patients } \\
\text { with PRT } \\
(n)\end{array}$ & $\begin{array}{l}\text { Images } \\
\text { during } \\
\text { FU after } \\
\text { PRT }(n)\end{array}$ & $\begin{array}{l}\text { Recurrent } \\
\text { RCC }(n)\end{array}$ & $\begin{array}{l}\text { INI1P } \\
\text { with } \\
\text { recurrent } \\
\operatorname{RCC}(n)\end{array}$ & $\begin{array}{l}\text { Patients } \\
\text { undergoing } \\
\text { TCI for } \\
\text { recurrent } \\
\text { RCC }(n)\end{array}$ & $\begin{array}{l}\text { INI1P } \\
\text { undergoing } \\
\text { TCI for } \\
\text { recurrent } \\
\text { RCC }(n)\end{array}$ & $\begin{array}{l}\text { Patients } \\
\text { alive with } \\
\text { NED after } \\
\text { TCI for } \\
\text { recurrent } \\
\text { RCC }(n)\end{array}$ & $\begin{array}{l}\text { INI1P alive } \\
\text { with NED } \\
\text { after TCI } \\
\text { for } \\
\text { recurrent } \\
\text { RCC }(n)\end{array}$ \\
\hline Low risk & 806 & 8986 & 65 & 138 & 29 & 310 & 17 & 529 \\
\hline Intermediate risk & 497 & 5560 & 108 & 51 & 34 & 164 & 11 & 505 \\
\hline High risk & 309 & 2787 & 163 & 17 & 29 & 96 & 4 & 697 \\
\hline Total & 1612 & 17333 & 336 & 52 & 92 & 188 & 32 & 542 \\
\hline
\end{tabular}

$\mathrm{RCC}=$ renal cell carcinoma; PRT $=$ presumed radical treatment; FU = follow-up; INI1P = images need to identify one patient; TCI $=$ treatment with curative intent; $\mathrm{NRD}=$ no evidence of disease 
Click here to download high resolution image

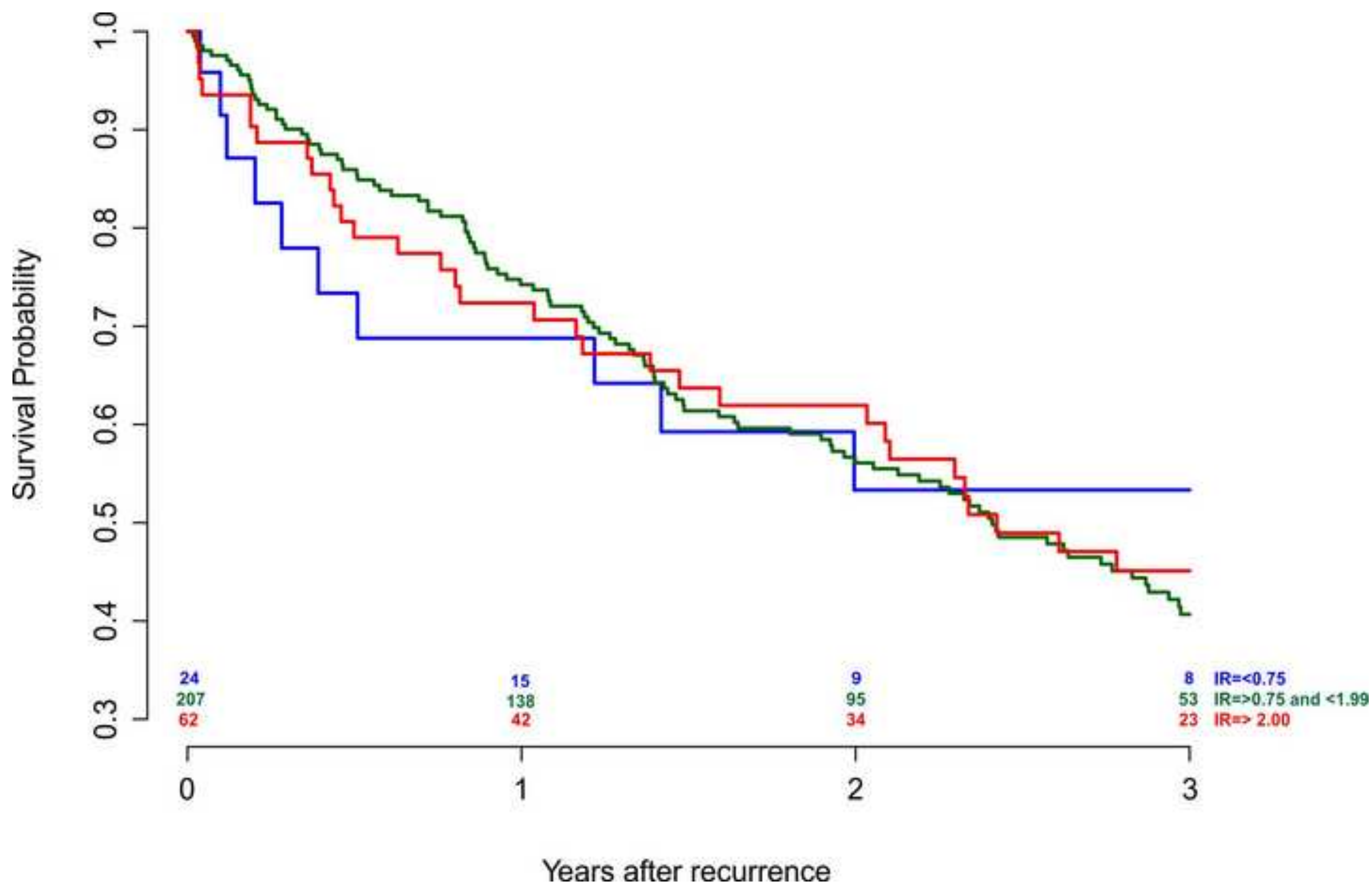

